\title{
Elementos del discurso del profesor que favorezcan el aprendizaje en las aulas universitarias
}

\section{Elements of the teacher's speech that promote learning at the university classrooms}

Morales Hidalgo Paulina

Pontificia Universidad Católica del Ecuador, Ecuador

Autor para correspondencia: pmorales048@puce.edu.ec

Fecha de recepción: 15 de diciembre de 2017 - Fecha de aceptación: 26 de febrero de 2018

\section{Resumen}

Los procesos de comunicación en las aulas universitarias son de vital importancia, el discurso del profesor, con su hacer didáctico, favorece o no el aprendizaje del adulto pues intervienen una serie de códigos que no se limitan a los lingüísticos, además del objeto de estudio a ser decodificado. Esto origina la investigación y análisis semiótico de este discurso para describir los elementos que allí intervienen, es una investigación cualitativa, con un paradigma socio crítico con un método Hermenéutico Dialéctico. Los resultados de la investigación identificaron los distintos componentes a los que se enfrentan los adultos aprendices en los procesos comunicativos del aula, que sirvieron de insumo para describir los elementos del discurso del profesor utilizando a la semiótica como un instrumento fundamental de interpretación y decodificación de los distintos códigos sociolingüísticos.es una investigación, con un enfoque introspectivo vivencial, que no es estático representa una realidad ontológica totalmente dinámica y cambiante

Palabras claves: discurso; profesor; semiótica; objeto de estudio; aprendizaje

\begin{abstract}
The communication processes in the university classrooms are of vital importance, the discourse of the teacher, with its didactic doing, favors or not the learning of the adult as a series of codes intervene that are not limited to the linguistic ones, besides the object of study to be decoded This originates the investigation and semiotic analysis of this discourse to describe the elements that intervene there, it is a qualitative research, with a critical socio-paradigm with a Dialectical Hermeneutical method. The results of the research identified the different components that the adult learners face in the communicative processes of the classroom, which served as an input to describe the elements of the teacher's discourse using semiotics as a fundamental instrument of interpretation and decoding of the different codes sociolinguísticos.es a research, with an experiential introspective approach, which is not static represents a fully dynamic and changing ontological reality.
\end{abstract}

Key words: speech; teacher; semiotics; object of study; learning 


\section{Introducción}

Esta investigación se enmarca en una concepción de la didáctica de las ciencias dedicada al análisis e investigación de la multi-dimensionalidad de los procesos de enseñanza y aprendizaje, los constructos de esta investigación son: el discurso del profesor, dentro del cual está inmerso el objeto del conocimiento; los adultos como aprendices, la didáctica, y la semiótica como disciplina que determina los contextos (situacional, lingüístico y mental), para poner en común o en signos convencionales, tanto para el profesor como para el alumno, el objeto del conocimiento.

Es por ello necesario definir este proceso pedagógico como un ajuste de la semiosis cognitiva, según García (2001) consiste en determinar críticamente la eficacia de mensajes, si entre determinados mensajes y sus correspondientes interpretaciones, se da o correspondencia, así como para constatar si determinada selección y contextualización de entidades (signos, producto, idea, comportamiento) habrán de generar en el intérprete (alumno) la misma significación que pretendió atribuirle su productor; que en el ámbito educativo sería el profesor. A ello habría que añadir: ¿cómo funciona este proceso y qué elementos intervienen en el discurso pedagógico?

Por el carácter singular, asimétrico e intencional en la forma de comunicación didáctica de cada asignatura, es necesario buscar indicadores que permitan la interpretación a partir del análisis semiótico del discurso del profesor para definir sus elementos; evidenciando esta situación en este estudio en situaciones reales de la Universidad Católica Andrés Bello, Venezuela

\section{Explorar la Importancia del problema}

Tradicionalmente se ha considerado el proceso de comunicación en el aula como algo mecánico y como un medio para presentar información, sin embargo este discurso es el que realmente propicia o no el aprendizaje, aun cuando se hable en una lengua convencional, no debe olvidarse que "los signos lingüísticos son totalmente arbitrarios" (Ferdinand de Saussure, citado por Arrellano.1979, pp. 149) y como tal "los procesos de interpretación en el alumno son de un signo que evoca otro y otro y otro hasta llegar a un proceso de semiósis ilimitada" (Peirce, citado por Greimas, 1990, pp. 43), lo que potencia el valor y la importancia del discurso pedagógico y su estudio como uno de los elementos fundamentales en el modelo del aprendizaje.

Esta investigación generará nuevos conocimientos ya que se justifica por el cuerpo de teorías que la orientan como son la del discurso en su contexto, los códigos que se expresan en lo cognitivo; el análisis estructural del discurso; además de la teoría del análisis semántico y la de los actos de habla en las que se vislumbra el ámbito propicio para el establecimiento de un nuevo método multidisciplinario en el análisis semiótico del discurso.

A nivel personal y profesional representa la posibilidad de aplicar la experiencia vivida durante quince años como profesora y aplicar los procesos semióticos al análisis pedagógico y cómo ocurre el fenómeno de significación e interpretación en el alumno, descubrir y plasmar lo experimentado con una base epistemológica que va más allá de la experiencia y las 
observaciones al alumno en su proceso de aprendizaje; en su necesidad de internalizar ese nuevo objeto del conocimiento para hacerlo parte de su interpretante e interrelacionarlo con su vida, permitirá un gran aporte a la comprensión del fenómeno semiótico y de aprehensión del conocimiento en el aula.

Desde la perspectiva metodológica el trabajo se justifica ya que permitirá establecer las variables que están incidiendo en el discurso pedagógico para determinar el aprendizaje o no del adulto. Este estudio permitirá crear un espacio para ir más allá de simples análisis de contenido con respecto a la caracterización específica de los distintos tipos de discurso utilizados en el aula con los que trabajan los adultos en calidad de aprendices, en su interacción con el profesor ya que se proporcionará herramientas analíticas básicas para implementar un modelo que interprete y reconstruya cualquier proceso de aprendizaje en su base y funcionamiento empíricos y en su vinculación con procesos socioculturales y de poder más amplios.

Mediante el análisis del discurso es posible identificar y analizar los mecanismos discursivos utilizados para persuadir al interlocutor, y descifrar los mensajes expresados superficialmente; o los mensajes ocultos, o no, a través de matrices que analizan sintagmas, signos lingüísticos, categorías gramaticales y sintaxis, esto a nivel general del discurso más aún en el ámbito educativo donde el objetivo de persuasión de todo acto de habla se ve potenciado en educación por el objetivo mismo del proceso educativo.

Su justificación práctica está determinada por el impacto social que los referidos constructos puedan generar al solucionar el problema que constituye la carencia de un análisis semiótico de los discursos pedagógicos y el hacer didáctico del profesor y su incidencia en el adulto como aprendiz.

\section{Revisión y Análisis Teórico}

García, W. y Martín, M (2013) desarrollaron una investigación sobre la Hermenéutica y pedagogía. La práctica educativa en el discurso sobre la educación, Esta investigación determinó el discurso pedagógico en el contexto cognitivo de los estudiantes. El método utilizado fue hermenéutico asumiendo los paradigmas epistemológicos cualitativo y cuantitativo con una modalidad de campo, desde la perspectiva discursiva, se denota en el contexto cognitivo de los estudiantes la existencia de implicaciones que producen malentendidos porque no se activan los conocimientos en forma apropiada, lo cual, incide en los criterios de confirmación y verdad que ocurren en los encuentros de enseñanza. En tal sentido, los estudiantes confrontan dificultades para descifrar con su propio código el objeto del estudio para darle significado al texto del profesor sin perder la conciencia de lo expresado por él.

Pena y Calzadilla (2006) investigaron los aportes teóricos, de Gadamer, Martínez y Ricoeur quienes coinciden en que la cualidad del objeto del conocimiento, es el resultado de la dialéctica y la hermenéutica aplicada de manera creativa y constructiva. Esta manera de abordar el discurso del profesor por parte del alumno permite su participación dialéctica para lograr descifrar al objeto del conocimiento; sin embargo, se convierte simplemente en el consenso de lo comprendido por el profesor y consensuado por los estudiantes. 
Mora (2006) en su investigación que aporta con la dimensión semiótica: titulada Aproximación a la inteligencia colectiva en donde plantea que la semiótica en el pensamiento científico es una ciencia en formación permanente, en cuanto disciplina destinada al estudio de los signos y de la dinámica de los sistemas de signos en la vida de la sociedad. Según este estudio conviene tejer y destejer las distintas dimensiones que supone el discurso del fenómeno humano, dentro del ámbito educativo; a partir de interrelaciones (sintaxis) entre los signos que conforman el discurso pedagógico, con definidas implicaciones dentro de un contexto sociocultural, histórico-social (pragmática), es decir, en el estudio de la relación entre los signos y sus usuarios.

Por otro lado, el adulto aprendiz participa de una actividad didáctica específica, con diferentes niveles de comunicación. El primero es el intraindividual e intrapsicológico que permite revisar sus conocimientos y referentes. Posteriormente, por intercambio con otros, se pasa a un nivel interindividual, que provoca la circulación del mensaje que se explicita y comparte en el aula.

Se produce el fenómeno de semiosis cognitiva García (2001), entendiéndose la cognición como un fenómeno que implica tanto la cognición personal como la cognición social, las creencias y los objetivos, así como las valoraciones y las emociones, junto con cualquier otra estructura, representación o proceso mental o memorístico que haya intervenido en el discurso y en la interacción en el aula, ya que el profesor trae al aula y recontextualiza,el objeto de estudio, creando situaciones para su desarrollo. Dependerá de la ocurrencia de dichas situaciones de clase que el aprendiz logre re-des-contextualizarlos Brousseau, (1996), es decir, construir significados y procesos personales. En esto radica precisamente el efecto del poder de un discurso, en su capacidad de poner en marcha un mecanismo de creencias, una adhesión, o un nuevo patrón o paradigma para ver las cosas.

\section{Pregunta de investigación y coherencia con el paradigma de investigación}

A lo largo de la historia, los científicos e investigadores al desarrollar estudios, o tratar de explicar la realidad, adoptan una posición de cómo van a abordar el objeto de investigación y para ello surgen los diversos paradigmas de investigación. Los paradigmas suelen identificarse en base a la forma en que tratan de dar respuesta, a algunas preguntas que surgen a distintos niveles de la realidad (Barrera Morales, 2004, pp 45) por paradigma se entiende como una perspectiva epistémica bajo la cual es apreciada una realidad cualquiera; paradigma significa "del lado de", "en la perspectiva de", "bajo la óptica de", y se refiere a una manera determinada de ver la realidad, una forma de actuar o una particular manera de ver el todo (el holos).

En esta investigación el paradigma adoptado es el Sociocrítico, que se fundamenta en la crítica social con un carácter reflexivo, considera que el conocimiento se construye por intereses que parten de las necesidades de los grupos, utiliza la autoreflexión y el conocimiento para que cada cual tome conciencia del rol que le corresponde, para ello se propone la descripción e interpretación de la realidad ya que la pregunta de investigación es:

¿Cómo funciona el proceso comunicativo en el aula y qué elementos intervienen en el discurso pedagógico? 
Entre las características más importantes del paradigma sociocrítico según Alvarado (2008), aplicado al ámbito de la educación, se encuentra la adopción de:

1. Una visión global y dialéctica de la teoría educativa

2. La aceptación de una visión democrática del conocimiento, así como de los procesos implicados en su elaboración, y

3. De una visión particular de la teoría del conocimiento y de sus relaciones con la realidad y la práctica.

Esta postura sociocrítica adoptada en la presente investigación permite crear una visión para comprender la semiosis que se logra en el aula en la construcción del conocimiento, según Habermas (1989) la sociedad es un mundo de significados y sentidos que deben ser analizados e interpretados para las mejoras sociales correspondientes. Esta dimensión de la comprensión entre los seres humanos dio paso al desarrollo de las ciencias hermenéuticas.

En este orden de ideas Habermas (1989) distingue dos funciones mediadoras de la ciencia social crítica entre lo teórico y lo práctico; a saber, una dimensión instrumental y la otra comunicacional. La dimensión instrumental origina una interacción social basada en intereses comunes para el logro de un objetivo, por ejemplo, un grupo de personas que quieran lograr algo en común requieren de intereses comunes y un adecuado cálculo para lograr el éxito.

La dimensión comunicacional en cambio promueve una interacción basada en procesos de interpretación para que los individuos participantes realicen una comprensión compartida de la misma y generen un consenso. Si esta segunda dimensión se lleva al ámbito educativo los individuos participantes serían los adultos aprendices que dentro del aula generan un consenso compartido del objeto de estudio para su correcta interpretación, ya que estos aprendices en el aula comparten un mundo simbólico que garantiza que se le otorgue validez a su proceso dialéctico y por supuesto a su aprendizaje.

Aquí se resume el paradigma sociocrítico en el que se basa el diseño de un modelo de análisis semiótico del discurso del profesor que favorezca su hacer didáctico y el aprendizaje del adulto como aprendiz, para comprender cómo funcionan los procesos dialécticos y discursivos en el aula, por lo que se considera pertinente y relevante; desde el plano Ontológico en la presente investigación se describe una realidad múltiple ya que el ámbito educativo jamás es estático, su dinámica es permanente y primordialmente dialéctica.

El abordaje epistemológico es subjetivista, (Ortiz ,1994, pp.49) "no hay diferenciación entre sujeto y objeto del conocimiento. Ambos se funden en una sola entidad epistemológica" ya que entre el objeto de investigación y el sujeto investigador hay una estrecha relación, ambos entran en el juego de la subjetividad del investigador y la de los sujetos participantes, pues están inmersos en la realidad ontológica.

\section{Método}


El método hermenéutico dialéctico fue el utilizado en esta investigación, Dilthey (citado por Martínez ,1989), define la hermenéutica como el proceso mediante el cual se conoce la vida psíquica con la ayuda de signos sensibles que son su manifestación.

La hermenéutica permite descubrir los significados, interpretar lo mejor posible las palabras, los escritos, los textos y los gestos, así como cualquier acto u obra, pero conservando su singularidad en el contexto de cual forma parte. En este sentido, una realidad social no puede cuadricularse en un plano cartesiano y tabla porcentual (Martínez, M. 1989), puesto que la sociedad es dinámica, es esa realidad múltiple y ontológica de significantes y significados que constituyen un sintagma en permanente construcción.

Para ilustrar este estudio se tomó como muestra a 35 estudiantes del primer año de Educación Preescolar e Integral de la Universidad Católica Andrés Bello, las cátedras seleccionadas en la Escuela de Educación, fueron las que menos promedio tuvieron durante los últimos 3 años y son: 2005 al 2008, son Psicología General y del Aprendizaje; Sociología de la Educación; Lengua y Comunicación; Historia Universal y Sistema Educativo Venezolano.

Los datos se analizaron considerando la investigación cualitativa que reconoce la subjetividad de los sujetos como parte constitutiva de su proceso indagador, por lo tanto la confiabilidad y la validez con un método hermenéutico dialéctico está dada porque los hallazgos que se comprueban a través de los diversos medios e instrumentos de investigación están en relación con sus objetivos, así en las investigaciones cualitativas sobre todo las referidas al análisis de contenidos, según Barrera, J. (2010) al desarrollo conceptual del análisis del contenido siguen las propuestas hermenéuticas que orientan hacia el enriquecimiento del discurso y su literalidad para lograr proponer categorías semánticas de significación que dan riqueza interpretativa a los textos.

\section{$1^{o}$ Paso Determinación del objetivo que se persigue:}

El objetivo de esta investigación es definir a través del análisis semiótico del discurso del profesor que favorezca su hacer didáctico y el aprendizaje del adulto como aprendiz, (Martínez, 1997, pp 126) es necesario tomar en cuenta dos criterios fundamentales para este quehacer, ¿Qué

voy a estudiar?, y ¿Por Qué?, según este autor "deben ser investigaciones en el área del comportamiento humano y es deseable que los objetivos propuestos estén relacionados con el conocimiento, el desarrollo y la promoción de las potencialidades de la persona.

Otro de los criterios es el metodológico en donde las dimensiones sean lo más concretas posibles, por ello ha de apoyarse en una revisión documental exhaustiva, sobre la aceptación de ciertas teorías para abordar el objetivo, "En tal caso conviene aclararlo de manera explícita al desarrollar el marco teórico que debe preceder a toda investigación respetable. (Martínez 1997, pp.126)

\section{$2^{o}$ Paso: Elección de la muestra a estudiar}

La determinación de la muestra es un paso fundamental ya que a través de ella se busca resultados que puedan generalizarse y depende de la naturaleza del conocimiento a alcanzar, por 
ello "no es verdad que cuanto mayor sea la muestra, mayor será la generalizabilidad de los datos o estructura obtenible" (Martinez, 1997, pp.128,).

\section{Población y Muestra}

La muestra descansa en el principio de que las partes representan al todo y, por tal, refleja las características que definen la población de la que fue extraída, lo cual indica que es representativa. La población que compete a esta investigación se encuentra en las aulas universitarias ya que aquí se presentan formas complicadas, desde el punto de vista estudiantil, en el procesamiento del objeto de estudio en el proceso semiótico ya que hay desplazamientos contextuales, el adulto como aprendiz no comprende textos sintácticamente rigurosos, se pierde al encontrar oraciones compuestas del lenguaje, las subordinaciones y el discurso del profesor determinan su aprendizaje.

Por lo que para ilustrar este estudio se tomó como muestra a 35 estudiantes del primer año de Educación Preescolar e Integral de la Universidad Católica Andrés Bello, Venezuela.

Las cátedras seleccionadas en la Escuela de Educación de la UCAB Guayana no escapan al problema plateado durante los últimos 3 años: 2005 al 2008, son Psicología General y del Aprendizaje; Sociología de la Educación; Lengua y Comunicación; Historia Universal y Sistema Educativo Venezolano.

\section{$3^{a}$ Paso: Elección de los instrumentos y Técnicas de Recolección}

Según Martínez (1997) los procedimientos y técnicas se eligen por el nivel de adecuación al fin perseguido y serán acorde a cada investigación, sin embargo, es conveniente ubicar ciertos criterios a tener en cuenta:

El primer criterio es que no debe deformar o distorsionar la realidad que se estudia, se deben registrar los acontecimientos por los actores del proceso investigado.

El segundo criterio es que la misma realidad debe ser observada varias veces o desde varias perspectivas, conviene esquematizarlas en anotaciones extraídas de los mismos actores del proceso observado, lo que permite lograr testimonios reales. Cada instrumento se elaboró tomando en cuenta la definición conceptual de cada constructo que orientó parte de las entrevistas para la recolección de la información.

\section{Instrumentos}

En esta investigación se elaboraron varios instrumentos para abordar los distintos discursos de los profesores y se determinó la frecuencia en los fenómenos observados tomando en cuenta criterios semiológicos.

A continuación, se presentan en forma de tabla la definición conceptual de cada constructo que orientó la construcción de las matrices de análisis, constituyendo un punto de partida para la recolección de la información. Para abordar el constructo del Discurso del Profesor se hizo un 
análisis simbólico, ya que según Barrera Morales (2004, pp.47) dicho análisis “estudia los aspectos de carácter representacional presentes en un estudio cualquiera", para esto todo signo es capaz de ser interpretable en un contexto significacional, llámese esto un cuadro, un discurso, un edificio, una clase.

Una vez determinado el constructo se elaboraron estas preguntas que se discutieron con los estudiantes a los que previamente se les explicó que era necesario saber cómo están comprendiendo el discurso el profesor y cómo es su proceso de comprensión en cada materia seleccionada, se aplicó estas preguntas por cada asignatura.

Tabla 1. Constructo: Discurso del Profesor

\begin{tabular}{|c|c|c|}
\hline Constructo & Definición conceptual & $\begin{array}{c}\text { Orientación de las preguntas a desarrollar } \\
\text { con la muestra internacional }\end{array}$ \\
\hline $\begin{array}{l}\text { Discurso } \\
\text { del profesor }\end{array}$ & $\begin{array}{l}\text { Para efectos de esta investigación el constructo } \\
\text { discurso se utiliza en el amplio sentido del } \\
\text { acontecimiento comunicativo que incluye la } \\
\text { interacción conversacional, todo el vocabulario } \\
\text { del profesor, los textos escritos y también los } \\
\text { gestos (prosémica y kinésica, llamados } \\
\text { subcódigos) asociados, las imágenes utilizadas } \\
\text { por el profesor y cualquier otra dimensión o } \\
\text { significación semiótica o multimedia. }\end{array}$ & $\begin{array}{l}\text { ¿Qué tipo de signos lingüísticos apoya la } \\
\text { creación del discurso del profesor? ¿Cómo es } \\
\text { evaluado su discurso por el profesor? ¿Cómo } \\
\text { fluye la comunicación en clase? ¿Qué } \\
\text { conocimientos implica? ¿Qué tipo de } \\
\text { palabras utiliza el profesor repite mucho } \\
\text { alguna? ¿Cómo se viste el profesor? ¿Qué } \\
\text { otros aspectos refiere el profesor en clase que } \\
\text { no sean objeto de estudio? }\end{array}$ \\
\hline
\end{tabular}

Nota: Los pasos fundamentales para este tipo de análisis son la determinación de los signos y símbolos. En el caso que ocupa esta investigación los signos lingüísticos y los no lingüísticos utilizados por el profesor determinan la comprensión del objeto de estudio.

Tabla 2. Constructo: Semiótica

\begin{tabular}{|c|c|c|}
\hline Constructo & Definición conceptual & $\begin{array}{c}\text { Orientación de las preguntas a desarrollar con } \\
\text { la muestra internacional }\end{array}$ \\
\hline Semiótica & $\begin{array}{l}\text { Para esta investigación la semiótica permite } \\
\text { identificar las distintas señales, signos y códifos } \\
\text { de comunicación lingüísticos. Es el proceso de } \\
\text { comprensión en la transformación del nuevo signo } \\
\text { en la mente del adulto aprendiz graciasd a la } \\
\text { comprensión del objeto del conocimiento dado } \\
\text { por el profesor, a través de un discurso, producto } \\
\text { de un proceso de análisis y percepción que será } \\
\text { determinado por la comprensión. }\end{array}$ & $\begin{array}{l}\text { ¿Entiende al profesor? Explique si su respuesta es } \\
\text { negativa o positiva ¿Cómo presenta conocimiento } \\
\text { que le ofrece el profesor? ¿Qué tipo recursos } \\
\text { utiliza el profesor para la explicación en su clase? } \\
\text { Definir los signos no lingüísticos que utiliza el } \\
\text { profesor. ¿Las oraciones que utiliza el profesor } \\
\text { son complicadas se entienden? ¿Qué texto, } \\
\text { láminas utiliza el profesor para presentar el objeto } \\
\text { de estudio? Análisis Paradigmático y } \\
\text { sintagmático con la matriz de sustancia y forma? }\end{array}$ \\
\hline
\end{tabular}

Nota: Con la Semiótica se utilizó el análisis paradigmático y sintagmático de autores basados en Ferdinand de Saussure como Hjemslev, quienes ilustran el proceso de análisis de los signos lingüísticos a través de una matriz, llamada de sustancia y forma, paralela al significante y significado de Saussure.

Tabla 3 Constructo el Hacer Didáctico 


\begin{tabular}{|c|c|c|}
\hline Constructo & Definición conceptual & $\begin{array}{c}\text { Orientación de las preguntas a desarrollar con la } \\
\text { muestra internacional }\end{array}$ \\
\hline $\begin{array}{l}\text { Hacer } \\
\text { Didáctico }\end{array}$ & $\begin{array}{l}\text { Para efectos de esta investigación el } \\
\text { Hacer didáctico la calidad de } \\
\text { interacción con los aprendices lo que } \\
\text { permite definir el significado cada } \\
\text { objeto de estudio una realidad } \\
\text { manifestada, para describirla y en } \\
\text { algunos casos reconocerla. }\end{array}$ & $\begin{array}{c}\text { ¿Usa muchos gestos el profesor kinésicos? ¿Cómo utiliza } \\
\text { el espacio en el aula el profesor? Proxémicos ¿Cómo fluye } \\
\text { la comunicación en clase? ¿Qué conocimientos implica? } \\
\text { ¿Perlocutivo todo a la defensiva? ¿Cómo utiliza los colores } \\
\text { el profesor? ¿Irlocutivo tono áspero del hablante? ¿Le } \\
\text { distrae su manera de vestir? Código de bienes ¿Cuándo } \\
\text { escucha al profesor es coherente lo que él dice? Cohesión, } \\
\text { conectores, coherencia }\end{array}$ \\
\hline
\end{tabular}

Nota: El constructo del Hacer didáctico del profesor es el conjunto de medios que hacen posible el reconocimiento de un objeto del conocimiento tal como aparece durante y después de su descripción, a través de signos lingüísticos o no utilizados por el profesor que permiten conocer.

Tabla 4. Constructo: Adulto

\begin{tabular}{|c|c|c|}
\hline Constructo & Definición conceptual & $\begin{array}{c}\text { Orientación del criterio para la } \\
\text { selección de la muestra } \\
\text { intencional }\end{array}$ \\
\hline Adulto & $\begin{array}{c}\text { Para esta investigación se tomaron en cuenta los postulados de } \\
\text { Adam (1977). El hombre se hace adulto no por la yuxtaposición } \\
\text { de un aspecto de su personalidad sobre los otros, sino por un } \\
\text { proceso de integración de sus diferentes estados tanto } \\
\text { biológicos, psicológicos y mentales, así como ergólógicos, } \\
\text { sociales y jurídicos. H10 }\end{array}$ & $\begin{array}{l}\text { Según Adam (1977) Participación } \\
\text { voluntaria del adulto. Respeto } \\
\text { mutuo. Espíritu de colaboración. } \\
\text { Reflexión y acción. Reflexión } \\
\text { crítica. Auto-dirección }\end{array}$ \\
\hline
\end{tabular}

Nota: El adulto aprendiz es aquel que tiene autonomía no puede entenderse en el sentido meramente psicológico, sino que debe abarcar todos los aspectos del ser humano que lo van conformando.

\section{Resultados y Discusión}

Para explicar el logro del objetivo de esta investigación es necesario integrar los componentes de un discurso pedagógico en sus diferentes ámbitos de análisis tanto paradigmático como sintagmático, Habermas (1989) define a los actantes que están presentes en el aula y son los que establecen un proceso de relaciones que va más allá de lo meramente comunicativo.

Los profesores para explicar y abordar la asignatura se enfrentan a un discurso propio del objeto de estudio que una vez reinterpretado formará un signo en la mente del adulto aprendiz para luego presentárselo a los estudiantes; este nuevo signo está impregnado de códigos sociales y sociolingüíticos que entran en juego y jamás serán imparciales ni abandonarán al profesor cuando se ubique frente a sus alumnos en el proceso aprendizaje.

Los componentes que entran en juego en el proceso hermenéutico desarrollado a través del discurso del profesor son:

$\checkmark$ Objeto de estudio

$\checkmark$ Campos de experiencia 
$\checkmark$ Referente o contexto de la comunicación

$\checkmark$ Hechos cognitivos

$\checkmark$ Códigos Sociolingüísticos

$\checkmark$ Marco del conocimiento

Los procesos de aprendizaje que se facilitan en las aulas se centran en la comunicación que posibilita el deseo por comprender tanto del profesor como del adulto aprendiz y esto sucede porque ambos comparten en el aula un mismo objeto o signo capaz de ser decodificado y reinterpretado acorde a cada uno de sus propios códigos tanto individuales y convencionales, por ello la semiosis ilimitada, en la que un signo una vez interpretado a través del proceso de la semiosis crea otro signo que es potencialmente interpretado nuevamente, lo que resume el proceso del aprendizaje..

Por ello, a veces, se producen malos entendidos con los discursos del profesor por parte de los estudiantes por el lenguaje utilizado por éste en sus actos comunicativos. A ello se suma, la complejidad y distancia social entre los profesores y alumnos que aunque comparten un mismo salón y están ligados por el interés de un mismo objeto de estudio, pertenecen a contextos totalmente diferentes

Todos estos componentes a su vez tienen sus propias extensiones y conforman un todo interviniente entre sus componentes e independientes además determinados por el contexto en donde se realizan.

Una vez trabajados los cuatro momentos de la investigación, se observa que se ha dado respuesta al objetivo de identificar los elementos del discurso del docente, aquí se hace una presentación más completa de las conclusiones del trabajo con las respectivas dimensiones que se desprenden de este análisis: integrar los componentes de un discurso pedagógico en sus diferentes ámbitos de análisis paradigmático y sintagmático; los discursos analizados no son meras abstracciones salidas de manera improvisada del profesor son el resultado de la interpretación que, individual y colectivamente ha realizado.

Los profesores para la explicación y el abordaje de la asignatura, que está en sus manos explicar, también se enfrentan a un discurso propio del objeto de estudio que una vez reinterpretado se formará un signo en su mente para luego presentárselo a los estudiantes. Este nuevo signo está impregnado de códigos sociales y sociolingüíticos que entran en juego y jamás serán imparciales ni abandonarán al profesor cuando se ubique frente a sus alumnos en el proceso aprendizaje

Por lo tanto, el profesor y el adulto aprendiz están propensos a que en el uso de esos juegos del lenguaje se asuman movimientos de ruptura y distanciamiento, porque en esa combinación sintagmática no siempre hay convencionalidad con el aprendiz que a su vez debe llevar ese discurso a su comprensión en un proceso de decodificación. Todos estos componentes a su vez tienen sus propias extensiones y conforman un todo interviniente entre su componente e independientes además determinados por el contexto en donde se realizan.

\section{Conclusión}


El discurso pedagógico es una relación sintagmática y paradigmática del lenguaje en constante dinámica, que se evidencia a través de significantes o expresiones que evocan significados o contenidos para definir un objeto de estudio que ha sido previamente inferenciado por el profesor.

Los elementos que subyacen dentro de este discurso no son conscientes ni para el mismo profesor que sin darse cuenta no sólo transmite el objeto de estudio interpretado, sino que a través de este discurso pedagógico va inmersa su ideología, su historia, su educación, su entorno y visón del mundo. Los elementos que son componentes de este constructo son:

Objeto de estudio, Referente, Campos de experiencias, o contexto de la comunicación, Hechos cognitivos, Códigos Sociolingüísticos y Marco del conocimiento.

El referente es la realidad sobre la cual se produce la comunicación y por lo tanto es generadora de significado, el referente ubica en un mundo real y posible y es cultural o social, y también puede ubicar un mundo ficticio en un mundo irreal y fragmentado sin que por ello carezca de sentido.

Otro de los componentes del discurso es el objeto de estudio al que se refiere el profesor en cada asignatura y es el objeto y razón de ser de su trabajo hermenéutico algunos autore $\mathrm{s}$ como Gadamer (1994) lo sitúan en tres niveles de interpretación:

El primero: formas del lenguaje (léxico, gramática, prosodia, que el adulto aprendiz debe decodificar

El segundo nivel: el contexto de la frase (intenciones del hablante, conocimientos compartidos); aquí cabe la manera cómo se dicen las cosas que tiene un significado tácito y

El tercer nivel tiene que ver con los códigos ideológicos. Es importante aclarar que el signo sociolingüístico es dinámico y tríadico.

El campo experiencia comprende el conjunto de ideas, valoraciones creencias, opiniones intenciones, cultura, los psicólogos llaman a esto la maleta personal de cada uno de nosotros que al interactuar se pone en contacto con otras maletas en el caso educativo las de los adultos aprendices, el contexto de la comunicación es dónde se da el proceso comunicativo, las aulas pueden generar discursos de poder, por la disposición de los pupitres y la estructura del salón.

Los Hechos Cognoscitivos tiene que ver con la Memoria a corto y a largo plazo, ya que ambos actúan para apoyar al profesor en el procesamiento de la información en el momento de los actos de habla, la de corto plazo es sólo para un hecho comunicativo preciso y la memoria a largo plazo es la que permite tener la información para otras oportunidades.

Códigos Sociolingüísticos son los que permiten la formación de los sintagmas visuales, de bienes y todas las combinaciones que utiliza el profesor para presentar el objeto de estudio, todos estos componentes se entretejen y se solapan en un solo discurso. Es necesario comprender 
que cuando el profesor está en un salón de clases lleva inmerso todos estos elementos en su discurso y si se quiere crear un modelo sistémico cuyas partes interactúan y se justifican en función de los elementos que la componen no se pueden aislar como partes determinantes en la presentación del objeto de estudio.

El profesor debe comprenderse a sí mismo saber que está elaborando una historia en sus alumnos a través de su discurso que obviamente está generado dentro del referente o contexto educativo por lo que se convierte en un discurso de poder pues en el aula se legitiman saberes.

\section{Bibliografía}

Adam, Félix, (1997). Algunos Enfoques Sobre Andragogía. Universidad Nacional Experimental Simón Rodríguez. Caracas, Venezuela,

Alvarado y García (2008). Características más relevantes del paradigma socio crítico, aplicación en la investigación educativa, UPEL, Caracas.

Arellano Fernando, (1977). Historia de la Lingüística, Bajo el signo del estructuralismo, UCAB, Caracas.

Barrera, M. (2010). Sistematización de experiencias y generación de teorías. Caracas: SYPAL.

Barreras Morales, Marcos (2004). Modelos Epistémicos en investigación. Caracas: SYPAL, 100 pp.

Brousseau, G. (1996) Utilidad práctica de los enfoques teóricos para la organización conducción de las actividades didácticas, Argentina FAMAF-UNC.

Gadamer Hermenéutica, (1994). Verdad y método, Madrid, Tomo II, ediciones sígueme Salamanca.

García José David, (2001). Para Una Semiótica De La Intersubjetividad En La Comunicación Profesor-Alumno, Enfoque Greimasiano de la relación didáctica, Universidad De Lima Facultad de Comunicación.

García, W. y Martín, M. (2013) Hermenéutica y pedagogía. La práctica educativa en el discurso sobre la educación, revista Pulso, №.36, mayo, 55-78.

Greimas; Courtes y otros (1990), Semiótica, Biblioteca románica hispánica. Editorial Gredos, Madrid,

Habermas, J. (1989) Teoría de la Acción Comunicativa: Complementos y Estudios Previos. Madrid: Cátedra. 1989

Martínez M. (1989) El método hermenéutico-dialéctico en las ciencias de la coducta, Anthropos Venezuela. 
Martínez, M. (1997)..El paradigma emergente: hacia una nueva teoría de la racionalidad científica, México: Trillas.

Mora, P. (2006) Aproximación a la inteligencia colectiva, Espéculo. Revista de estudios literarios. Universidad Complutense de Madrid, $\mathrm{N}^{\circ} .16$, noviembre.

http://www.ucm.es/info/especulo/numero16/int_cole.html

Ortiz, JR (1994).Paradigmas de la Investigación Educativa. Revista Educativa y Ciencias Humana. No.3. pp. 151 - 164. Universidad Nacional Abierta. Caracas. Venezuela.

Pena, J y Calzadilla, R. (2006) Lo cualitativo del discurso pedagógico en la dialéctica hermenéutica. Sapiens. Revista Universitaria de Investigación, vol. 7, núm. 1, junio, pp. 181-202

Pena, José y Calzadilla, Ramón, (2006) Lo cualitativo del discurso pedagógico en la dialécticahermenéutica. vol.7, SAPIENS, Junio 